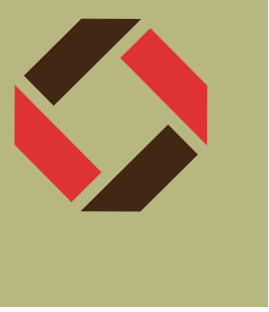

\title{
An art/science collaboration benefits nanotechnology, medicine, and art
}

\author{
By Lori A. Wilson
}

ive years ago, an artist and a scien-
tist met at an art exhibition, spurring a collaboration that resulted in a new kind of art, a new kind of outreach, and a new kind of sensor for cancer.

Houston-based visual artist Joseph Cohen was conducting his first solo exhibition in an art gallery in New York. Cohen mixes his own paints using raw pigment and varnish to create layered paintings designed to present different textures and colors when you view them from different angles. Each work contains approximately 30 different shades and up to 150 painted layers. Cohen's art practice focuses on "medium specificity" and how a work of art can serve to elucidate humans' relationship with the physical world (see Figure 1).

Daniel Heller, Head of the Cancer Nanomedicine Laboratory at Memorial Sloan Kettering Cancer Center in New York City visited the gallery, where he saw Cohen's work and recognized his approach as reminiscent of that of a materials scientist (see Figure 2).
Heller, admittedly not a student of the art world, asked Cohen, "So your art is really the paint, not the art?"

"Joseph was kind to entertain my exceptionally naive questions about his work," Heller said. Their conversation resulted in an invitation to Heller's laboratory the next day, where Heller introduced Cohen to the properties of singlewalled carbon nanotubes (SWCNTs).

SWCNTs exhibit unique optical properties, including highly photostable near-infrared fluorescence. Many different structural isomers of SWCNTs result in multiple, separable species with different emission wavelength bands from the 900-1400 $\mathrm{nm}$ range and beyond. "These properties are exceptional and allow us to develop new imaging probes and sensors, but few people know about them," Heller said. "I thought that if we can take these colors and make them accessible to an audience through art, it would be great STEM outreach."
Heller's laboratory, which uses SWCNTs to develop fluorescent biosensors for cancer research and diagnostics, worked with Cohen to develop SWCNTs into bright near-infrared paints. Together, they figured out how to make SWCNTs "paintable" by incorporating them into the right binder material that did not compromise their near-infrared emission. They then had to figure out how to image the paintings, initially using Heller's preclinical imaging instrument built to image mice, where they could only test mouse-sized paintings.

"I wanted to use carbon nanotubes to allow people to experience something 'invisible' - a range of colors that they wouldn't normally have access to," Cohen said.

"Without any formal research lab training, Joseph was able to jump from the art world into nanomaterials research, and from abstract art to photophysics," Heller said. "I realized that his talents could help the public experience many types of materials and scientific concepts, and I also realized that we needed more help from the nanocarbons field to get our project up and running."

Heller i n v i t e d Cohen to a Electro-
Figure 1. "MSP 001 (Multi-Spectrum-Painting), 2019" painting by Joseph Cohen. Separated carbon nanotubes, surfactant, binder, tracer dyes, and doped fluorescent and phosphorescent gouache paints on linen $(24 \times 24$ in.). Left, visible spectrum; right, nearinfrared fluorescence emission. 


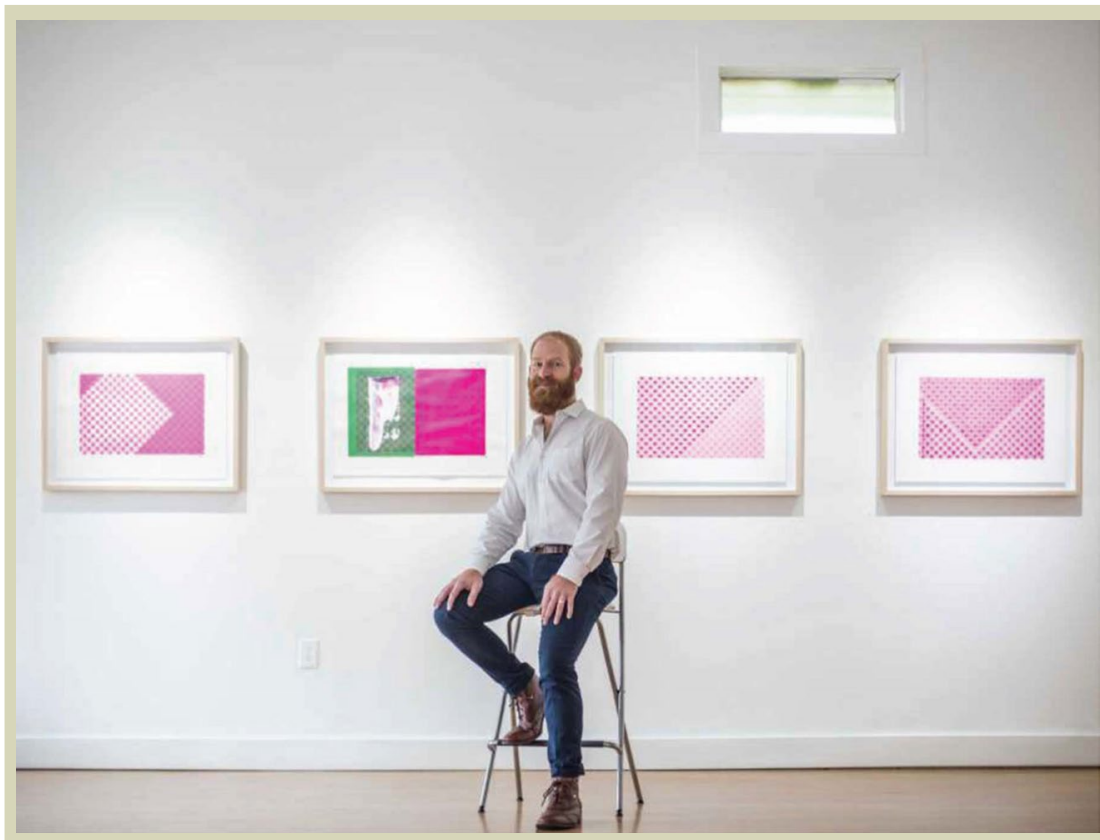

Figure 2. Joseph Cohen illustrates nanomaterials' unique characteristics through art.

chemical Society meeting to introduce him to more nanocarbon researchers. There, Cohen met Bruce Weisman from Rice University, one of the first people to study carbon nanotube (CNT) fluorescence. Weisman worked with Cohen to enable him to illuminate and image much larger paintings using a custom-built light-emitting diode setup.

“Weisman's knowledge of the optical properties of CNTs is unparalleled, and his insights into how to most efficiently engineer a device that would enable us to both excite the material and image the artwork proved to be invaluable," said Cohen. The work was then brought to Montreal to be photographed using an InGaAs camera at the company Photon, etc.

The collaborators realized another benefit of SWCNTs in visual art is that they are stable and unreactive, making them "archival materials" in artist parlance, and they could potentially be used to prove authenticity.

CNTs have made news in the art world before; Vantablack, a vertically aligned array of nanotubes, was developed to absorb up to $99.965 \%$ of visible light and is often dubbed the "blackest black." Controversy arose because it was licensed exclusively to the well-known artist Anish Kapoor, prompting a backlash from some artists.

The exclusive license to one artist was due, in part, according to the company, because it did not have the bandwidth to produce enough material for all artists to use, bringing up another issue that Heller and Cohen are hoping to address. "Because of their strength and electrical properties, carbon nanotubes are an ideal material for many applications, such as structural composites and electronics, but their potential has not yet been fully realized," said Heller. This is partially because more work is needed to successfully engineer them for many applications. For instance, individual structural isomers of CNTs are difficult to isolate, and the separation process has not yet been scaled up commercially.

The different isomers of CNTs have different electronic and optical properties, and, when separated, they do not look black anymore to the naked eye; they exhibit different visible colors as pigments, as well as different emission wavelengths in the near-infrared.

Once the individual CNT structures are separated, they can be used for archival pigments of many colors as well as near-infrared fluorescent paints with many different infrared "colors." "But scientists and engineers could certainly use these isolated nanotube materials for electronics and as multiplexed nearinfrared optical probes and sensors," said Heller. "Part of this outreach was to show people how beautiful and useful the materials can be, and hopefully it could help prompt commercial interest in making them available to us all."

The art/science collaboration also had direct benefits to cancer research in Heller's lab. "Once we made the nearinfrared paints with Joseph, we realized that we could use that knowledge to make sensor paints for diagnostic applications," Heller said.

Heller's lab made a nanosensor paint to detect microalbuminuria, a condition where small amounts of albumin build up in the urine. Microalbuminuria is an important clinical marker of diabetes, hypertension, and cancer. CNTs, encapsulated by a polymer, act as the transduction element that produces a shift in nanotube emission in the presence of albumin. Embedded into a paintable binder material, the sensor paint can detect albumin in clinical urine samples and be applied to nearly any surface. The work was published in 2019 in Nature Communications. ${ }^{1}$

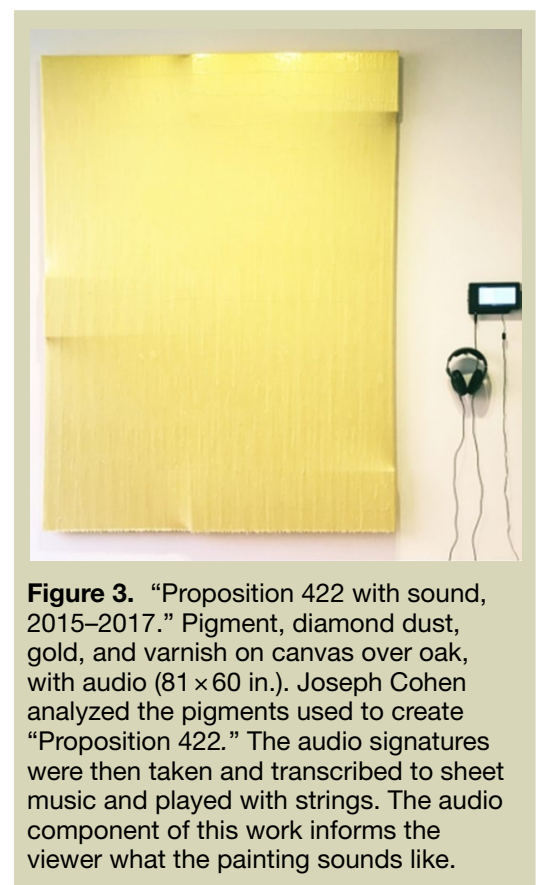


"We initially thought that the art would shed a little light on our science," Heller said. "But this outreach actually helped our lab to shine a light on cancer."

Inspired by his initial art/science collaboration, Cohen has expanded this work into different materials and art forms. Collaborating with James Tour at Rice University, Cohen developed paintings by converting paper into graphene using a high-powered laser. The work was published in $2019 .{ }^{2}$ Cohen also worked with Christian Boada in Ennio Tasciotti's lab, at the Houston Methodist Research Institute at Houston Methodist Hospital, to attempt to address what a painting sounds like (see Figure 3). "For

\section{References}

1. J. Budhathoki-Uprety, J. Shah, J.A. Korsen, A.E. Wayne, T.V. Galassi, J.R. Cohen, J.D. Harvey, P.V. Jena, L.V. Ramanathan, E.A. Jaimes, D.A. Heller, Nat. Commun. 10, 3605 (2019)

2. Y. Chyan, J. Cohen, W. Wang, C. Zhang, J.M. Tour, ACS Appl. Nano Mater. 2(5), 3007 (2019)

this project, we analyzed individual pigments through Fourier-transform infrared spectroscopy. The data and waveforms that were collected then went into an algorithm that generated 'color chords' that could be used to create both sheet music and paintings," said Cohen.
"Over time, our ability to learn from one another, expand boundaries of thought, and share our diverse set of experiences hope to not only push the boundaries of art, but also add a level of creativity to science and engineering labs for which true discovery can incubate," said Cohen.

We welcome comments and feedback on this article via email to Bulletin@mrs.org.

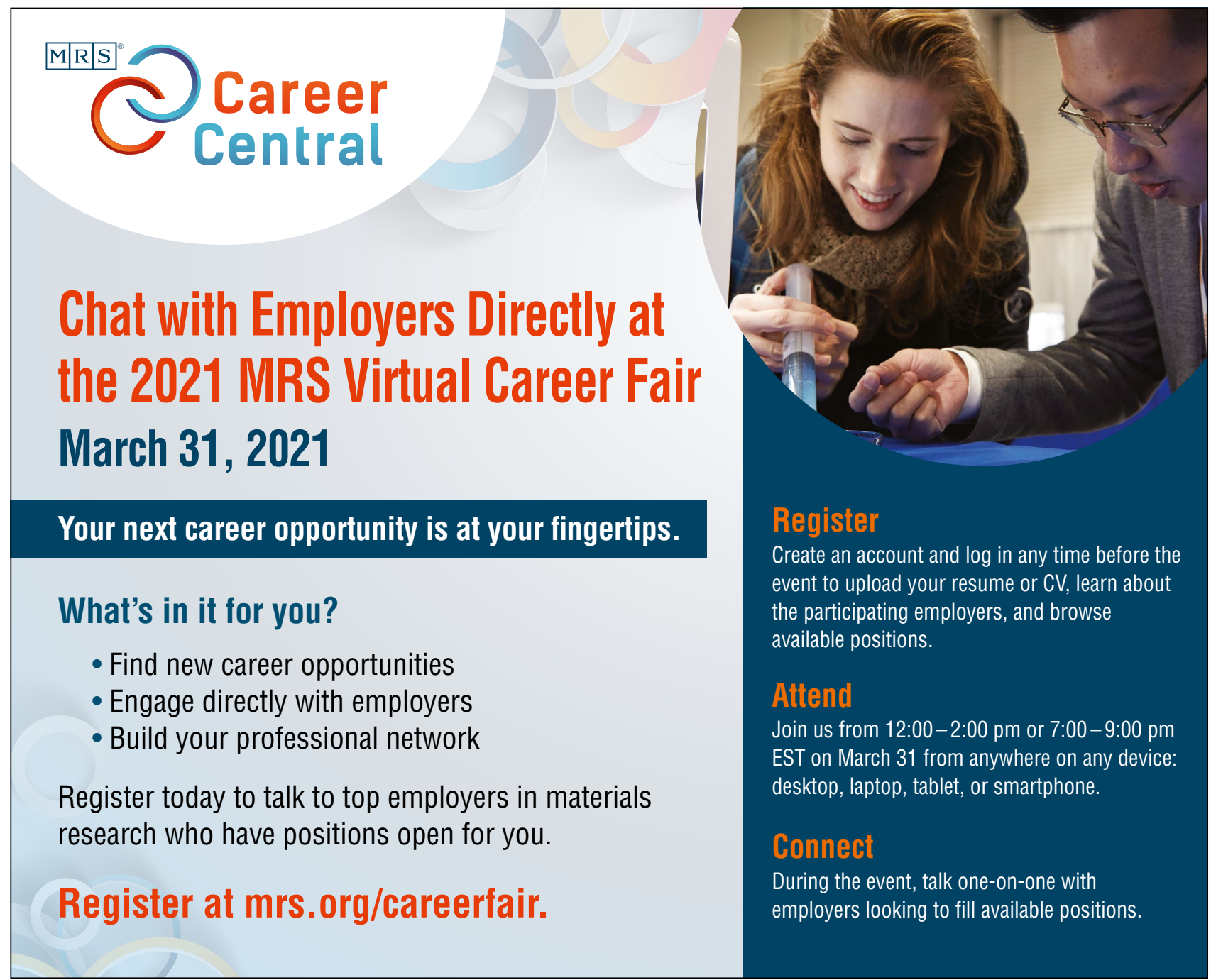

